\title{
Novel Prescribed Performance Control Scheme for Flexible Hypersonic Flight Vehicles with Nonaffine Dynamics and Neural Approximation
}

\author{
Yong Liu $\mathbb{D},{ }^{1}$ Gang $\mathrm{Li} \mathbb{D}^{1},{ }^{1}$ Yuchen $\mathrm{Li}^{2}$ and Yahui $\mathrm{Wu}{ }^{3}$ \\ ${ }^{1}$ Air and Missile Defense College, Air Force Engineering University, Xi'an 710051, China \\ ${ }^{2}$ Air Traffic Control and Navigation College, Air Force Engineering University, Xi'an 710051, China \\ ${ }^{3}$ Radar Sergeant School, Air Force Early Warning Academy, Wuhan 430345, China \\ Correspondence should be addressed to Yong Liu; romeo1881021@163.com
}

Received 27 July 2020; Revised 18 August 2020; Accepted 20 January 2021; Published 10 February 2021

Academic Editor: Jacopo Serafini

Copyright (@) 2021 Yong Liu et al. This is an open access article distributed under the Creative Commons Attribution License, which permits unrestricted use, distribution, and reproduction in any medium, provided the original work is properly cited.

\begin{abstract}
This study develops a novel neural-approximation-based prescribed performance controller for flexible hypersonic flight vehicles (HFVs). Firstly, a new prescribed performance mechanism is exploited, which develops new performance functions guaranteeing velocity and altitude tracking errors with small overshoots. Compared with the existing prescribed performance mechanism, it has better preselected transient and steady-state performance. Then, the nonaffine model of HFV is decomposed into a velocity subsystem and an altitude subsystem. A prescribed performance-based proportional-integral controller is designed in the velocity subsystem. In the altitude subsystem, the model is expressed as a nonaffine pure feedback form, and control inputs are derived from neural approximations. In order to reduce the amount of computation, only one neural network approximator is used to approximate the subsystem uncertainties, and an advanced regulation algorithm is applied to the devise adaptive law for neural estimation. At the same time, the complex design process of back-stepping can be avoided. Finally, numerical simulation results are presented to verify the efficiency of the design.
\end{abstract}

\section{Introduction}

Hypersonic flight vehicles (HFVs) fly in near space and fly at speeds greater than five times the speed of sound, including aerospace vehicles with horizontal takeoff and landing, reentry vehicles, hypersonic cruise missiles, and many other types of flight vehicles [1-3]. Compared with existing spacecraft, near space hypersonic flight vehicles can reach global targets in a short time and have great potential in military and civilian applications. Designing a rational control system is not only one of the core technologies of HFVs but also a challenging subject. During the high-speed flight of HFVs, the drastic changes of aerodynamic and aerothermal characteristics will change many parameters of the vehicles, so it is very important to study the steady-state performance of the control system. At the same time, under the constraints of narrow flight corri- dors, improving the transient performance of the system is also one of the problems to be solved.

Most of the research results of control systems for HFVs focus on the longitudinal model of HFV due to the fact that HFVs themselves are characterized by fast time variation, strong nonlinearity, strong coupling, and uncertainty, and the longitudinal model is complex enough. In order to save fuel and keep the control system stable, HFV should try to avoid lateral maneuver [4]. Meanwhile, many advanced methods are used in control systems for HFVs, including robust control $[5,6]$, sliding mode control $[7,8]$, backstepping control $[2,6]$, and intelligent control.

In [9], a self-scheduled robust decoupling control law is designed for HFVs; the simulation results verify the reliability of the method. Aiming at the parameter perturbation and external disturbance existing in HFVs, a robust controller is designed in [10], which combines with non- 
linear disturbance observer and back-stepping control method. However, as the order of the system increases, the differential expansion problem occurs. In [11], a dynamic surface robust controller is designed for the input constraints and parameter uncertainties of HFV. According to the longitudinal model of HFV, [12] introduces a nonlinear disturbance observer and designs a novel integrated sliding mode controller. When the system has external disturbances, chattering, and model uncertainties, it can ensure stable tracking. In $[13,14]$, in order to achieve quasicontinuous and continuous switching of control inputs, a high-order sliding mode control scheme is studied. In [15], a fuzzy back-stepping control law is proposed. Through the back-stepping method and the fuzzy logic system, stable tracking is achieved. Under strict assumptions, the altitude dynamics of HFVs can be rewritten as a strict-feedback system. And with the new back-stepping control scheme, only the final control law is required to be implemented in the new back-stepping control method. In [16], the longitudinal model of HFV is expressed by the Takagi-Sugeno (T-S) fuzzy system, and a $H_{2} / H_{\infty}$ tracking control law is designed. The above works are mainly aimed at the stability performance of HFV system. If we limit the transient performance of the system, it is necessary to adjust the controller parameters instantaneously. However, due to the numerous and complex parameters in the controller, it is difficult to ensure that the multiple performance indices of the system can meet the prescribed requirements at the same time.

Bechlioulis et al. first propose a prescribed performance control strategy $[17,18]$. The main idea of the prescribed performance control scheme is to introduce the performance function to standardize corresponding indices of the system. By constructing the error conversion function, an original "constrained" system is transformed into an equivalent "unconstrained" one. In [19], considering a class of nonlinear systems with unknown state variables, a fuzzy state observer is introduced to reconstruct the unknown state variables, and a prescribed performance fuzzy back-stepping controller is designed. Similarly, [20] integrates prescribed performance with high-order extended state observer via dynamic surface control, which can estimate unknown state variables and eliminate involving errors. But in [17-20], the performance function needs to know the sign of the initial error. In [21], a prescribed performance control strategy is applied to the HFV control system; the model is simplified to a strictfeedback formulation. However, the design process of the control law is too cumbersome, and the initial value of the performance function is required to be large enough. In view of the above, a novel prescribed performance control scheme guaranteeing the tracking error with a small, even zero, overshoot is proposed. And the scheme is applied to the HFV model with nonaffine dynamics. The special contributions are summarized as follows:

(1) A novel prescribed performance function is proposed. Compared with $[21,22]$, the proposed prescribed performance mechanism has better transient performance, which guarantees that a small, even zero, overshoot can be imposed on tracking errors
(2) Compared with [23], the prescribed performance mechanism proposed in this paper does not simplify the key nonaffine dynamics

(3) In this paper, the total system uncertainties are approximated by a neural network approximator, and the adaptive law is designed by the norm estimation approach [24]. Compared with [25], the computational complexity is greatly reduced.

\section{Problem Formulation}

2.1. HFV Model Description. Considering the longitudinal model of HFV, Bolender and Doman propose the First Principle model [26]. Based on the First Principle model, Parker et al. neglect some weak coupling and slow dynamics in the model and establish a control-oriented longitudinal parameter fitting model of HFV [27].

$$
\begin{aligned}
\dot{V} & =\frac{T \cos (\theta-\gamma)-D}{m}-g \sin \gamma, \\
\dot{h} & =V \sin \gamma \\
\dot{\gamma} & =\frac{L+T \sin (\theta-\gamma)}{m V}-\frac{g}{V} \cos \gamma, \\
\dot{\theta} & =Q, \\
\dot{Q} & =\frac{M+\tilde{\psi}_{1} \ddot{\eta}_{1}+\tilde{\psi}_{2} \ddot{\eta}_{2}}{I_{y y}}, \\
\delta_{1} \ddot{\eta}_{1} & =-2 \zeta_{1} \omega_{1} \dot{\eta}_{1}-\omega_{1}^{2} \eta_{1}+N_{1}-\tilde{\psi}_{1} \frac{M}{I_{y y}}-\frac{\tilde{\psi}_{1} \tilde{\psi}_{2} \ddot{\eta}_{2}}{I_{y y}}, \\
\delta_{2} \ddot{\eta}_{2} & =-2 \zeta_{2} \omega_{2} \dot{\eta}_{2}-\omega_{2}^{2} \eta_{2}+N_{2}-\tilde{\psi}_{2} \frac{M}{I_{y y}}-\frac{\tilde{\psi}_{2} \tilde{\psi}_{1} \ddot{\eta}_{1}}{I_{y y}}
\end{aligned}
$$

where

$$
\left\{\begin{array}{l}
\delta_{1}=1+\frac{\tilde{\psi}_{1}}{I_{y y}} \\
\delta_{2}=1+\frac{\tilde{\psi}_{2}}{I_{y y}} \\
\tilde{\psi}_{1}=\int_{-L_{f}}^{0} \widehat{m}_{f} \xi \phi_{f}(\xi) \mathrm{d} \xi \\
\tilde{\psi}_{2}=\int_{0}^{L_{a}} \widehat{m}_{a} \xi \phi_{a}(\xi) \mathrm{d} \xi
\end{array}\right.
$$

$\phi_{f}(\cdot)$ and $\phi_{a}(\cdot)$ are structural mode shapes [26]. The geometry and force map of HFV model is shown in Figure 1.

The longitudinal model of HFV includes rigid body states and flexible states. The rigid body states are $\{V, h, \gamma, \theta, Q\}$, wherein, $V$ and $h$ represent the flight velocity and altitude and $\gamma$ and $\theta$ are the attitude angles of HFV, representing the flight path angle and the pitch angle, respectively. $Q$ represents the pitch rate, $m$ is the mass of the HFV, $r$ is the radial 


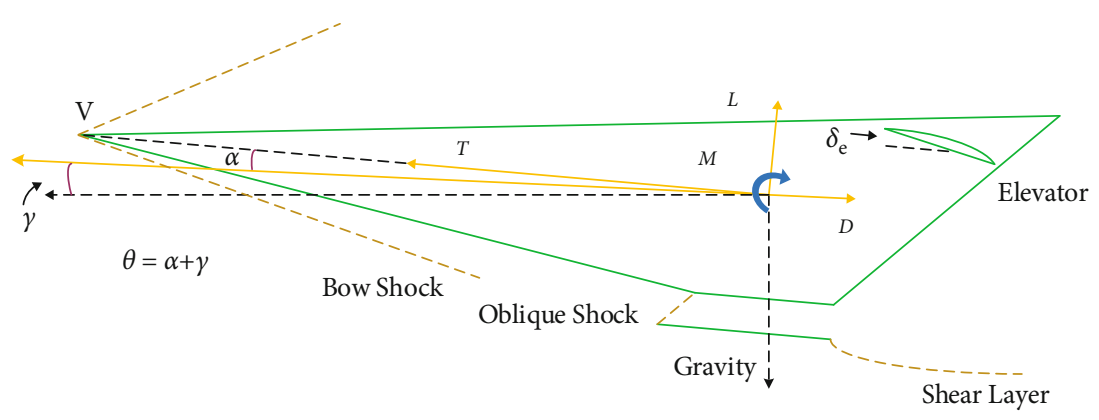

FIGURE 1: Geometry and force map of HFV model.

distance from the center of the earth, $I_{y y}$ is the moment of inertia about the pitch axis, and $\eta_{1}$ and $\eta_{2}$ are the flexible states. T, $D, L, M, N_{1}$, and $N_{2}$ represent the thrust, drag force, lift force, pitching moment, first generalized force, and second generalized force, respectively. Their fitting form is shown in

$$
\begin{aligned}
T & \approx C_{T}^{\alpha^{3}} \alpha^{3}+C_{T}^{\alpha^{2}} \alpha^{2}+C_{T}^{\alpha} \alpha+C_{T}^{0}, \\
D & \approx \bar{q} S\left(C_{D}^{\alpha^{2}} \alpha^{2}+C_{D}^{\alpha} \alpha+C_{D}^{\delta_{\mathrm{e}}^{2}} \delta_{\mathrm{e}}^{2}+C_{D}^{\delta_{\mathrm{e}}} \delta_{\mathrm{e}}+C_{D}^{0}\right), \\
L & \approx \bar{q} S\left(C_{L}^{\alpha} \alpha+C_{L}^{\delta_{\mathrm{e}}} \delta_{\mathrm{e}}+C_{L}^{0}\right), \\
M & \approx z_{T} T+\bar{q} S \bar{c}\left[C_{M, \alpha}^{\alpha^{2}} \alpha^{2}+C_{M, \alpha}^{\alpha} \alpha+C_{M, \alpha}^{0}+c_{\mathrm{e}} \delta_{\mathrm{e}}\right], \\
N_{1} & \approx N_{1}^{\alpha^{2}} \alpha^{2}+N_{1}^{\alpha} \alpha+N_{1}^{0}, \\
N_{2} & \approx N_{2}^{\alpha^{2}} \alpha^{2}+N_{2}^{\alpha} \alpha+N_{2}^{\delta_{\mathrm{e}}} \delta_{\mathrm{e}}+N_{2}^{0}, \\
C_{T}^{\alpha^{3}} & =\beta_{1}(h, \bar{q}) \Phi+\beta_{2}(h, \bar{q}), \\
C_{T}^{\alpha^{2}} & =\beta_{3}(h, \bar{q}) \Phi+\beta_{4}(h, \bar{q}), \\
C_{T}^{\alpha} & =\beta_{5}(h, \bar{q}) \Phi+\beta_{6}(h, \bar{q}), \\
C_{T}^{0} & =\beta_{7}(h, \bar{q}) \Phi+\beta_{8}(h, \bar{q}), \\
\bar{q} & =\frac{1}{2} \bar{\rho} V^{2}, \bar{\rho}=\bar{\rho}_{0} \exp \left(\frac{h_{0}-h}{h_{\mathrm{s}}}\right),
\end{aligned}
$$

where $\bar{\rho}$ represents the average air density and $\bar{q}$ represents the dynamic pressure of HFV. $h_{0}$ represents nominal altitude and $h_{\mathrm{s}}$ represents the altitude constant. $\bar{\rho}_{0}$ represents the air density at $h_{0}$. For more detailed definitions of the model variables and coefficients, we can refer to $[26,27] . \Phi$ and $\delta_{e}$ represent control inputs; furthermore, it is observed from the longitudinal model that the control inputs $\Phi$ and $\delta_{\mathrm{e}}$ do not occur explicitly in (1), (2), (3), (4), (5), (6), and (7). Since there is no actual actuator to control the two flexible states, and the two flexible states are also unmeasurable, in the controller, the flexible states are treated as unknown disturbances.

Remark 1. It can be seen from Equation (9) that the parameter fitting form of the drag force $D$ contains $\delta_{e}^{2}$, so from the perspective of the control inputs, the HFV model is nonaffine, if it is simply to simplify the nonaffine dynamics into affine dynamics, which will cause the loss of key dynamics, and make the simplified affine model control law partially invalid or a control failure.

2.2. Novel Prescribed Performance Mechanism. The primary task of the prescribed performance control mechanism is to design the prescribed performance function and then construct the error conversion function to ensure that the tracking error $e(t)$ can converge to an adjustable residual set with the expected convergence time and the maximum overshoot less than a designed value. The traditional form of performance function is shown in $[17,28]$

$$
\rho(t)=\left(\rho_{0}-\rho_{\infty}\right) e^{-l t}+\rho_{\infty} .
$$

Among them, $l \in R^{+}, \rho_{0} \in R^{+}$, and $\rho_{\infty} \in R^{+}$are parameters to be designed. According to the performance requirements, tracking error $e(t)$ needs to satisfy

$$
\left\{\begin{array}{l}
-\bar{m} \rho(t)<e(t)<\rho(t), e(0)>0, \\
-\rho(t)<e(t)<\bar{m} \rho(t), e(0)<0,
\end{array}\right.
$$

where $\bar{m}$ is the parameter to be designed and $0 \leq \bar{m} \leq 1$. According to the above analysis, in fact, we need to know the sign of $e(0)$ a priori, and according to the sign, we can choose the appropriate conditions in Equation (11), but the initial value of the tracking error is generally difficult to obtain. Therefore, in the process of control law design and stability analysis, a variety of cases need to be considered. The relationship between tracking error $e(t)$ and performance function $\rho(t)$ is clearly illustrated in Figure 2.

In this paper, we propose a novel formulation of performance as follows:

$$
\lambda_{L}(t)<e(t)<\lambda_{U}(t) .
$$

The novel performance functions $\lambda_{L}(t)$ and $\lambda_{U}(t)$ are constructed as

$$
\left\{\begin{array}{l}
x_{L}(t)=\left[\frac{\operatorname{sign}(e(0))-\iota_{L}}{\sinh (\delta t+\sigma)}\right]-\iota_{L} \rho_{\infty}, \\
\chi_{U}(t)=\left[\frac{\operatorname{sign}(e(0))+\iota_{U}}{\sinh (\delta t+\sigma)}\right]+\iota_{U} \rho_{\infty} .
\end{array}\right.
$$




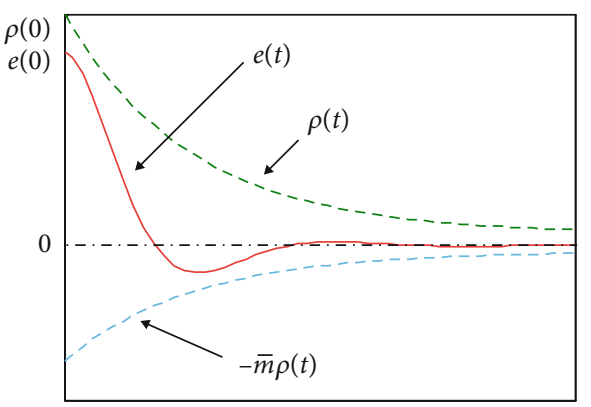

Time (s)

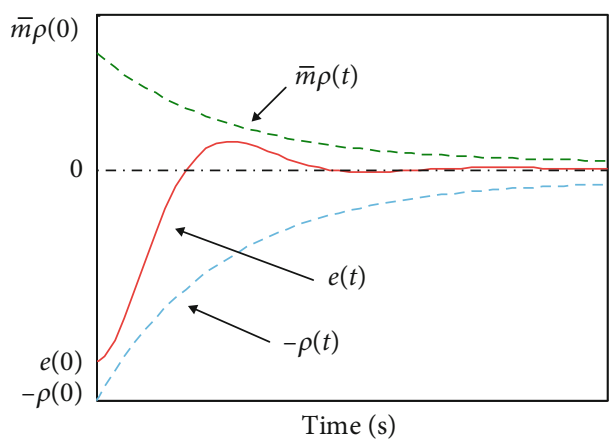

Figure 2: Graphical illustration of the traditional prescribed performance definition (11): (a) $e(0)>0$; (b) $e(0)<0$.

Taking the time derivative of (13),

$$
\left\{\begin{array}{l}
\dot{x}_{L}(t)=\frac{-\delta\left[\operatorname{sign}(e(0))-\iota_{L}\right] \cosh (\delta t+\sigma)}{[\sinh (\delta t+\sigma)]^{2}}, \\
\dot{\lambda}_{U}(t)=\frac{-\delta\left[\operatorname{sign}(e(0))+\iota_{U}\right] \cosh (\delta t+\sigma)}{[\sinh (\delta t+\sigma)]^{2}}
\end{array}\right.
$$

where $\iota_{L} \in R^{+}, \iota_{U} \in R^{+}, \delta \in R^{+}, \sigma \in R^{+}$, and $\rho_{\infty} \in R^{+}$are parameters to be designed.

Lemma 2 (see [17]). If the smoothing function $\rho(t)$ is a monotonically decreasing positive function, and $\lim _{t \rightarrow \infty} \rho(t)=\rho_{\infty}$, then $\rho(t)$ is a prescribed performance function.

According to Lemma 2,

$$
\left\{\begin{array}{l}
\lambda_{L}(0)=\left[\frac{\operatorname{sign}(e(0))-\iota_{L}}{\sinh (\sigma)}\right]-\iota_{L} \rho_{\infty}>-\iota_{L} \rho_{\infty}, \\
\lambda_{L}(\infty)=\left[\frac{\operatorname{sign}(e(t))-\iota_{L}}{\sinh (\delta t+\sigma)}\right]-\iota_{L} \rho_{\infty}=-\iota_{L} \rho_{\infty}, \\
\lambda_{U}(0)=\left[\frac{\operatorname{sign}(e(0))+\iota_{U}}{\sinh (\sigma)}\right]+\iota_{U} \rho_{\infty}>\iota_{U} \rho_{\infty}, \\
\lambda_{U}(\infty)=\left[\frac{\operatorname{sign}(e(t))+\iota_{U}}{\sinh (\delta t+\sigma)}\right]+\iota_{U} \rho_{\infty}=\iota_{U} \rho_{\infty} .
\end{array}\right.
$$

The new prescribed performance functions proposed in this paper satisfies Lemma 2. The performance constraints on tracking errors are shown in Figure 3.

Remark 3. Since the traditional prescribed performance function must obtain the initial error sign a priori, this disadvantage limits the operability of prescribed performance control. It can be seen from Figure 3 that the new prescribed performance functions proposed in this paper do not depend on the initial error; meanwhile, $\lambda_{L}(t)$ and $\lambda_{U}(t)$ can change the shape of the functions according to the different signs of $e(0)$. By selecting appropriate parameters, $e(t)$ can converge to the prescribed performance with a small overshoot.

\section{Preliminaries}

3.1. Error Transformation. Since it is cumbersome to design the control law directly for the prescribed performance functions, the functions need to be transformed. Define the transformed error as

$$
\mu(t)=\ln \left(\frac{\bar{\zeta}(t)}{1-\bar{\zeta}(t)}\right),
$$

where $\bar{\varsigma}(t)=\left[e(t)-\lambda_{L}(t)\right] /\left[\lambda_{U}(t)-\lambda_{L}(t)\right]$.

$$
e^{\mu(t)}=\frac{\bar{\varsigma}(t)}{1-\bar{\zeta}(t)} .
$$

Theorem 4. If the transformed error $\mu(t)$ is bounded, then $e$ (t) satisfies Equation (12).

Proof. The inverse of Equation (16) is

After converting Equation (17), we can get

$$
\bar{\zeta}(t)=\frac{e^{\mu(t)}}{1+e^{\mu(t)}}
$$

Since $\mu(t)$ is bounded, there must be a bounded constant $\mu_{M} \in R^{+}$such that $|\mu(t)| \leq \mu_{M}$. Equation (18) can be changed to

$$
0<\frac{e^{-\mu_{M}}}{1+e^{-\mu_{M}}}<\bar{\zeta}(t)<\frac{e^{\mu_{M}}}{1+e^{\mu_{M}}}<1 .
$$

Since $\bar{\varsigma}(t)=\left[e(t)-\lambda_{L}(t)\right] /\left[\chi_{U}(t)-\lambda_{L}(t)\right]$, there will be

$$
0<\frac{e(t)-\lambda_{L}(t)}{\lambda_{U}(t)-\lambda_{L}(t)}<1
$$

That is,

$$
\lambda_{L}(t)<e(t)<\lambda_{U}(t) .
$$

Remark 5. As can be seen from [17], if we can ensure that $\mu$ $(t)$ is bounded, then the tracking error $e(t)$ can meet the performance requirements. If the appropriate values are chosen for $\lambda_{L}(t)$ and $\lambda_{U}(t)$, it is guaranteed that $e(t)$ has satisfactory 

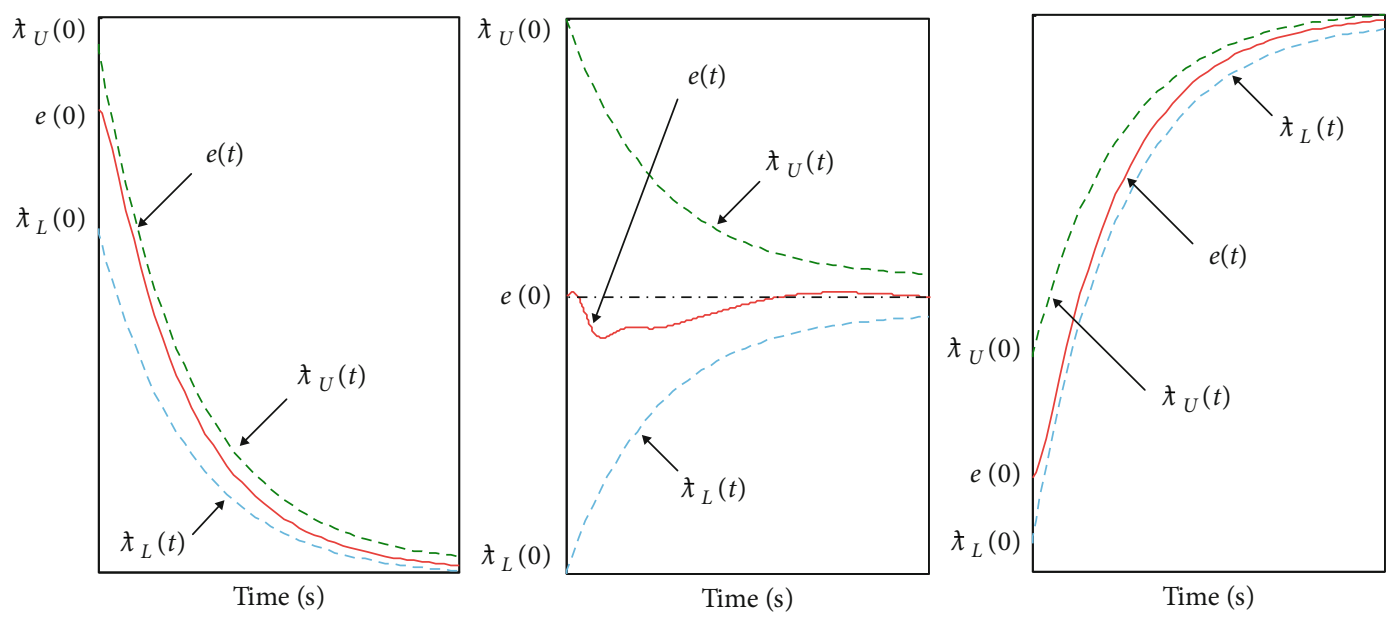

FIGURE 3: Graphical illustration of the novel prescribed performance definition (12): (a) $e(0)>0$; (b) $e(0)=0$; (c) $e(0)<0$.

dynamic performance and steady-state accuracy. In what follows, the controller will be explored using the transformed error $\mu(t)$ instead of the tracking error $e(t)$.

3.2. Neural Network Approximation. The RBF neural network (NN) has three layers: input layer, hidden layer, and output layer. The activation function of neurons in the hidden layer is the radial basis function. The array operation units that make up the hidden layer are called the hidden layer nodes. The RBF neural network can represent an input-to-output mapping, which is

$$
y=\mathbf{W}^{\mathrm{T}} \phi(\mathbf{X})
$$

where $\mathbf{X}=\left[x_{1}, x_{2}, \cdots, x_{n}\right]^{T} \in \mathbf{R}^{n}$ is the input vector, $\mathbf{W}=$ $\left[w_{1}, w_{2}, \cdots, w_{v}\right]^{T} \in \mathbf{R}^{v}$ represents the weight vector, and the output of the hidden layer is $\phi(\mathbf{X}), \quad \phi(\mathbf{X})=$ $\left[\phi_{1}(\mathbf{X}), \phi_{2}(\mathbf{X}), \cdots, \phi_{v}(\mathbf{X})\right]^{T} \in \mathbf{R}^{v}$. Each hidden layer contains a central vector $\mathbf{c} ; \phi_{j}(\mathbf{X})$ is the output of the $j$-th neuron of the hidden layer and can be expressed as

$$
\phi_{j}(\mathbf{X})=\exp \left(-\frac{\left\|\mathbf{X}-\mathbf{c}_{j}\right\|^{2}}{2 b_{j}^{2}}\right), \quad j=1,2, \cdots, v,
$$

where $b_{j}$ represents the width of the $j$-th neuron Gaussian function and $\mathbf{b}=\left[b_{1}, b_{2}, \cdots, b_{v}\right]^{T} \in \mathbf{R}^{v}$,

$$
\mathbf{c}=\left[\begin{array}{ccc}
c_{11} & \cdots & c_{1 v} \\
\vdots & \ddots & \vdots \\
c_{n 1} & \cdots & c_{n v}
\end{array}\right] .
$$

$n$ and $v$ are the dimensions of the input vector and the number of nodes, respectively. For any nonlinear continuous function $F(\mathbf{X})$, there must be an ideal weight vector $\mathbf{W}^{*}=$ $\left[w_{1}^{*}, w_{2}^{*}, \cdots, w_{v}^{*}\right]^{T} \in \mathbf{R}^{v}$, so that

$$
F(\mathbf{X})=\mathbf{W}^{* \mathrm{~T}} \mathbf{h}(\mathbf{X})+\varepsilon, \quad|\varepsilon| \leq \varepsilon_{M},
$$

where $\varepsilon \in R$ represents the approximation error of NN and $\varepsilon_{M} \in R^{+}$represents the upper bound of the approximation error. As long as we choose a large enough $v, \varepsilon_{M}$ can be arbitrarily small [29]. However, the online computational load will increase with a large $v$; it should be considered in the controller design [30].

\subsection{Nussbaum-Type Function}

Lemma 6 (see [31]). For function $\hbar(9)$, if the following properties hold

$$
\left\{\begin{array}{l}
\lim _{\beta_{0} \rightarrow \infty} \sup \frac{1}{\beta_{0}} \int_{0}^{\beta_{0}} \hbar(\vartheta) d \zeta=+\infty \\
\lim _{\beta_{0} \rightarrow \infty} \inf \frac{1}{\beta_{0}} \int_{0}^{\beta_{0}} \hbar(\vartheta) d \zeta=-\infty
\end{array}\right.
$$

Then, the function $\hbar(9)$ is a Nussbaum-type function.

Lemma 7 (see [32]). Define $Z_{0}(t) \geq 0$ on $\left[0, t_{1}\right)$ and $\hbar(\vartheta)$, if inequality holds

$$
Z_{0}(t) \leq \int_{0}^{t}\left(X_{0} \hbar(\vartheta)+1\right) \dot{\vartheta}(\tau) d \tau+H_{0}
$$

where $\lambda_{0}$ is nonzero and $H_{0}$ is a suitable constant, then $\hbar$ (9), $\int_{0}^{t}\left(\lambda_{0} \hbar(\vartheta)+1\right) \dot{\vartheta}(\tau) d \tau$, and $H_{0}$ are all bounded on $\left[0, t_{1}\right)$.

\section{Controller Design}

In what follows, we would present a prescribed performance control scheme with nonaffine dynamics and neural approximation, based on the transformed error (16), which leads to a simple controller, capable of guaranteeing the satisfaction of the constraint $|\mu(t)| \leq \mu_{M}$ and the boundedness of all other closed-loop signals.

4.1. Velocity Subsystem. The control goal of this section is to achieve stable tracking of $V \longrightarrow V_{\text {ref }}$ by designing an 
appropriate prescribed performance controller of velocity subsystem.

Since the velocity subsystem controller is relatively simple, based on the idea of [32], we design a prescribed performance proportional-integral (PI) controller without estimation parameters.

Velocity tracking error is defined as

$$
\tilde{V}=V-V_{\text {ref }}
$$
$\lambda_{U}^{V}(t)$

Select novel prescribed performance functions $\chi_{L}^{V}(t)$ and

$$
\left\{\begin{array}{l}
\rtimes_{L}^{V}(t)=\left[\frac{\operatorname{sign}(\tilde{V}(0))-\iota_{L}^{V}}{\sinh \left(\delta_{V} t+\sigma_{V}\right)}\right]-\iota_{L}^{V} \rho_{\infty}^{V}, \\
\chi_{U}^{V}(t)=\left[\frac{\operatorname{sign}(\tilde{V}(0))+\iota_{U}^{V}}{\sinh \left(\delta_{V} t+\sigma_{V}\right)}\right]+\iota_{U}^{V} \rho_{\infty}^{V},
\end{array}\right.
$$

where $\iota_{L}^{V} \in R^{+}, \iota_{U}^{V} \in R^{+}, \delta_{V} \in R^{+}, \sigma_{V} \in R^{+}$, and $\rho_{\infty}^{V} \in R^{+}$are parameters to be designed.

Define transformed error $\mu_{V}(t)$

$$
\mu_{V}(t)=\ln \left(\frac{\bar{\varsigma}_{V}(t)}{1-\bar{\varsigma}_{V}(t)}\right)
$$

where $\bar{\varsigma}_{V}(t)=\left[e(t)-\chi_{U}^{V}(t)\right] /\left[\chi_{U}^{V}(t)-\chi_{L}^{V}(t)\right]$.

Design the PI control law as

$$
\Phi=-\lambda_{V 1} \mu_{V}(t)-\lambda_{V 2} \int_{0}^{t} \mu_{V}(t) d \tau
$$

$\lambda_{V 1} \in R^{+}$and $\lambda_{V 2} \in R^{+}$are parameters to be designed. According to the PI controller, it can be seen that $\mu_{V}(t)$ is bounded. From the conclusion of Theorem 4 , if $\mu_{V}(t)$ is bounded, then the velocity tracking error $\tilde{V}$ satisfies

$$
\chi_{L}^{V}(t)<\tilde{V}<\chi_{U}^{V}(t)
$$

By selecting the appropriate $\chi_{L}^{V}(t)$ and $\chi_{U}^{V}(t), \tilde{V}$ can have good dynamic performance and steady-state accuracy.

4.2. Altitude Subsystem. In this section, we will design a prescribed performance controller for altitude subsystems (2), (3), (4), and (5) such that $h$ tracks its reference trajectory $h_{\text {ref }}$ with the altitude tracking error satisfying the preselected transient performance.

We define the altitude tracking error as

$$
\tilde{h}=h-h_{\text {ref }} .
$$

We select the prescribed performance functions of altitude subsystem as

$$
\left\{\begin{array}{l}
\lambda_{L}^{h}(t)=\left[\frac{\operatorname{sign}(\tilde{h}(0))-\iota_{L}^{h}}{\sinh \left(\delta_{h} t+\sigma_{h}\right)}\right]-\iota_{L}^{h} \rho_{\infty}^{h}, \\
\lambda_{U}^{h}(t)=\left[\frac{\operatorname{sign}(\tilde{h}(0))+\iota_{U}^{h}}{\sinh \left(\delta_{h} t+\sigma_{h}\right)}\right]+\iota_{U}^{h} \rho_{\infty}^{h} .
\end{array}\right.
$$

In Equation (34), $\iota_{L}^{h} \in R^{+}, \iota_{U}^{h} \in R^{+}, \delta_{h} \in R^{+}, \sigma_{h} \in R^{+}$, and $\rho_{\infty}^{h} \in R^{+}$are parameters to be designed. Transformed error $\mu_{h}(t)$ is defined as

$$
\mu_{h}(t)=\ln \left(\frac{\bar{\varsigma}_{h}(t)}{1-\bar{\varsigma}_{h}(t)}\right) .
$$

where $\bar{\varsigma}_{h}(t)=\left[e(t)-\lambda_{U}^{h}(t)\right] /\left[\lambda_{U}^{h}(t)-\lambda_{L}^{h}(t)\right]$.

Through feedback transformation, the control objective of the altitude subsystem is transformed into $\gamma \longrightarrow \gamma_{d}$ by selecting appropriate feedback control input $\delta_{e}$ [33].

The reference trajectory of $\gamma$ is chosen as

$$
\gamma_{\mathrm{d}}=\arcsin \left(\frac{-\lambda_{h} \mu_{h}(t)+\dot{h}_{\mathrm{ref}}+\dot{\rho}_{h}(t) \tilde{h} / \rho_{h}(t)}{V}\right) \text {, }
$$

where $\lambda_{h} \in R^{+}$is the parameter to be designed, $\rho_{h}(t)=$ $\sinh ^{-1}\left(\delta_{h} t+\sigma_{h}\right)+\rho_{\infty}^{h}$, and $\dot{\rho}(t)=-\delta_{h} \cosh \left(\delta_{h} t+\sigma_{h}\right) /[\sinh$ $\left.\left(\delta_{h} t+\sigma_{h}\right)\right]^{2}$.

Lemma 8 (see [32]). If $\gamma \longrightarrow \gamma_{d}$, then the dynamic response of $\mu_{h}(t)$ is

$$
\lambda_{h} \dot{\mu}_{h}(t)+\mu_{h}(t)=0 .
$$

Then, $\mu_{h}(t)$ must be bounded.

We further conclude that the prescribed performance of $\tilde{h}$ can be guaranteed according to Theorem 4. And the control goal of subsystem becomes $\gamma \longrightarrow \gamma_{d}$.

We define

$$
\left\{\begin{array}{l}
\chi_{1}=\gamma \\
\chi_{2}=\theta \\
\chi_{3}=Q
\end{array}\right.
$$

Express the other parts of the altitude subsystem (Equations (3), (4), and (5)) as nonaffine forms as follows:

$$
\left\{\begin{array}{l}
\dot{\chi}_{1}=\Gamma_{1}\left(\chi_{1}, \chi_{2}, \delta_{e}\right), \\
\dot{\chi}_{2}=Q \\
\dot{\chi}_{3}=\Gamma_{2}\left(\chi_{1}, \chi_{2}, \chi_{3}, \Phi, \delta_{e}\right),
\end{array}\right.
$$

where $\Gamma_{1}\left(\chi_{1}, \chi_{2}, \delta_{\boldsymbol{e}}\right)$ and $\Gamma_{2}\left(\chi_{1}, \chi_{2}, \chi_{3}, \Phi, \delta_{\boldsymbol{e}}\right)$ are completely unknown continuous functions; in the following section, we will transform the original nonaffine models (3), (4), and (5) into a norm output feedback formulation. 
We define $\omega_{1}=\chi_{1}=\gamma$ and $\omega_{2}=\dot{\omega}_{1}=\Gamma_{1}\left(\chi_{1}, \chi_{2}, \delta_{e}\right)$; applying (39), the time derivative of $\omega_{2}$ is derived as

$$
\begin{aligned}
\dot{\omega}_{2}= & \frac{\partial \Gamma_{1}\left(\chi_{1}, \chi_{2}, \delta_{e}\right)}{\partial \chi_{1}} \dot{\chi}_{1}+\frac{\partial \Gamma_{1}\left(\chi_{1}, \chi_{2}, \delta_{e}\right)}{\partial \chi_{2}} \dot{\chi}_{2}+\frac{\partial \Gamma_{1}\left(\chi_{1}, \chi_{2}, \delta_{e}\right)}{\partial \delta_{e}} \dot{\delta}_{e} \\
= & \frac{\partial \Gamma_{1}\left(\chi_{1}, \chi_{2}, \delta_{e}\right)}{\partial \chi_{1}} \Gamma_{1}\left(\chi_{1}, \chi_{2}, \delta_{e}\right)+\frac{\partial \Gamma_{1}\left(\chi_{1}, \chi_{2}, \delta_{e}\right)}{\partial \chi_{2}} \chi_{3} \\
& +\frac{\partial \Gamma_{1}\left(\chi_{1}, \chi_{2}, \delta_{e}\right)}{\partial \delta_{e}} \dot{\delta}_{e} \triangleq \psi\left(\chi_{1}, \chi_{2}, \chi_{3}, \delta_{e}\right) .
\end{aligned}
$$

Then, we define $\omega_{3}=\dot{\omega}_{2}=\psi\left(\chi_{1}, \chi_{2}, \chi_{3}, \delta_{e}\right)$; substituting (39) and (40), the time derivative of $\omega_{3}$ is derived as

$$
\begin{aligned}
\dot{\omega}_{3}= & \frac{\partial \psi\left(\chi_{1}, \chi_{2}, \chi_{3}, \delta_{e}\right)}{\partial \chi_{1}} \dot{\chi}_{1}+\frac{\partial \psi\left(\chi_{1}, \chi_{2}, \chi_{3}, \delta_{e}\right)}{\partial \chi_{2}} \dot{\chi}_{2} \\
& +\frac{\partial \psi\left(\chi_{1}, \chi_{2}, \chi_{3}, \delta_{e}\right)}{\partial \chi_{3}} \dot{\chi}_{3}+\frac{\partial \psi\left(\chi_{1}, \chi_{2}, \chi_{3}, \delta_{e}\right)}{\partial \delta_{e}} \dot{\delta}_{e} \\
= & \frac{\partial \psi\left(\chi_{1}, \chi_{2}, \chi_{3}, \delta_{e}\right)}{\partial \chi_{1}} \Gamma_{1}\left(\chi_{1}, \chi_{2}, \delta_{e}\right)+\frac{\partial \psi\left(\chi_{1}, \chi_{2}, \chi_{3}, \delta_{e}\right)}{\partial \chi_{2}} \chi_{3} \\
& +\frac{\partial \psi\left(\chi_{1}, \chi_{2}, \chi_{3}, \delta_{e}\right)}{\partial \chi_{3}} \Gamma_{2}\left(\chi_{1}, \chi_{2}, \chi_{3}, \Phi, \delta_{e}\right) \\
& +\frac{\partial \psi\left(\chi_{1}, \chi_{2}, \chi_{3}, \delta_{e}\right)}{\partial \delta_{e}} \dot{\delta}_{e} \triangleq \Upsilon\left(\chi, \Phi, \delta_{e}\right),
\end{aligned}
$$

with $\chi=\left[\chi_{1}, \chi_{2}, \chi_{3}\right]^{T} \in \mathbf{R}^{+}$.

Therefore, according to Equation (39), we can get a pure feedback nonaffine model.

$$
\left\{\begin{array}{l}
\dot{\omega}_{1}=\omega_{2}, \\
\dot{\omega}_{2}=\omega_{3}, \\
\dot{\omega}_{3}=\Upsilon\left(\chi, \Phi, \delta_{e}\right),
\end{array}\right.
$$

where $Y\left(\chi, \Phi, \delta_{e}\right)$ is a continuous unknown function.

In order to facilitate the next step of controller design, the implicit function theorem is derived.

Lemma 9 (implicit function theorem). If the implicit function $G(\emptyset, \sigma) \in \mathbf{R}^{m}$ and $\sigma \in \mathbf{R}^{n}$ satisfy the following conditions:

(1) $G(\emptyset, \sigma)=0$ is continuous in the region $D \subset \mathbf{R}^{m \times n}$ with $P_{0}\left(\varrho_{0}, \sigma_{0}\right)$ as the interior point

(2) $G\left(\varliminf_{0}, \sigma_{0}\right)=0$

(3) $(\partial G / \partial \omega)(\varrho, \sigma)$ and $(\partial G / \partial \sigma)(\varrho, \sigma)$ are continuous in region $D$

(4) $(\partial G / \partial \sigma)\left(\varrho_{0}, \sigma_{0}\right) \neq 0$

In the region $D$ of $P_{0}$, if $G(\oplus, \sigma)=0$, it can uniquely obtain a function $\sigma_{0}=g_{0}\left(\bigotimes_{0}\right)$ defined on the neighborhood $H \subset \mathbf{R}^{m}$ and get $G\left(\infty, g_{0}\left(\oplus_{0}\right)\right)=0$.
Remark 10. If the implicit function meets the above 4 conditions, $\sigma$ can be expressed as a continuous differentiable function of $₫$, i.e., $\sigma_{0}=g_{0}\left(\bigcup_{0}\right)$. And then, $G(\bowtie, \sigma)$ can also be viewed as a continuous differentiable function of $\emptyset$.

Assumption 11. It is assumed that $\left(\partial \Upsilon\left(\chi, \delta_{\mathrm{e}}\right)\right) /\left(\partial \delta_{\mathrm{e}}\right) \neq 0$.

Remark 12. Assumption 11 imposes the global controllability condition on Equation (42) and also is the satisfying condition of Lemma 9. Compared with previous studies [25, 34, $35]$, the proposed scheme based on the Nussbaum-type function does not need to guarantee that the control gain $(\partial \Upsilon$ ( $\left.\left.\chi, \delta_{\mathrm{e}}\right)\right) / \partial \delta_{\mathrm{e}}$ is strictly positive. The formulation of Equation (42) is more concise than Equation (39), and there is no need of complicated recursive design process of back-stepping and the virtual control law with a first-order filter, which greatly simplifies the complexity of the control law.

Define the tracking error $\tilde{\gamma}$ and the error function $f_{E}$ as shown in

$$
\left\{\begin{array}{l}
\tilde{\gamma}=\gamma-\gamma_{\mathrm{d}}=\omega_{1}-\gamma_{\mathrm{d}}, \\
f_{E}=\left(\frac{\mathrm{d}}{\mathrm{d} t}+\ell\right)^{3} \int_{0}^{t} \tilde{\gamma} \mathrm{d} \tau=\ddot{\tilde{\gamma}}+3 \ell \dot{\tilde{\gamma}}+3 \ell^{2} \tilde{\gamma}+\ell^{3} \int_{0}^{t} \tilde{\gamma} \mathrm{d} \tau,
\end{array}\right.
$$

where $\ell \in R^{+}$is the parameter to be designed.

The time derivative of $f_{E}$ is derived as

$$
\begin{aligned}
\dot{f}_{E} & =\ddot{\tilde{\gamma}}+3 \ell \ddot{\tilde{\gamma}}+3 \ell^{2} \dot{\tilde{\gamma}}+\ell^{3} \int_{0}^{t} \tilde{\gamma} \mathrm{d} \tau=\Upsilon\left(\chi, \delta_{\mathrm{e}}\right)-\ddot{\gamma}_{\mathrm{d}}+3 \ell \ddot{\tilde{\gamma}}+3 \ell^{2} \dot{\tilde{\gamma}}+\ell^{3} \tilde{\gamma} \\
& =\Upsilon\left(\chi, \delta_{\mathrm{e}}\right)-\Upsilon\left(\chi, \delta_{\mathrm{e}}^{*}\right)+\left[\Upsilon\left(\chi, \delta_{\mathrm{e}}^{*}\right)-\ddot{\gamma}_{\mathrm{d}}+3 e \tilde{\tilde{\gamma}}+3 \ell^{2} \dot{\tilde{\gamma}}+\ell^{3} \tilde{\gamma}\right] .
\end{aligned}
$$

Based on Assumption 11 and Lemma 9, there must be a $\delta_{\mathrm{e}}^{*}$ satisfying $r\left(\chi, \delta_{\mathrm{e}}^{*}\right)-\ddot{\gamma}_{\mathrm{d}}+3 \ell \ddot{\tilde{\gamma}}+3 \ell^{2} \dot{\tilde{\gamma}}+\ell^{3} \tilde{\gamma}=0$, then Equation (44) can be changed to

$$
\dot{f}_{E}=r\left(\chi, \delta_{\mathrm{e}}\right)-r\left(\chi, \delta_{\mathrm{e}}^{*}\right)
$$

We introduce the mean value theorem below.

Lemma 13 (mean value theorem). If the function $f(\mathbf{x}, y)$ satisfies the following conditions:

(1) $f(\mathbf{x}, y)$ has a derivative at each point of an open set $\mathbf{R}^{n} \times(a, b)$

(2) $f(\mathbf{x}, y)$ is continuous at both endpoints $y=a$ and $y=b$

Then, there is a point $\xi \in(a, b)$ such that

$$
\dot{f}(\mathbf{x}, \xi)=\frac{f(\mathbf{x}, b)-f(\mathbf{x}, a)}{b-a} .
$$


According to Lemma 13, we convert Equation (45) into

$$
\dot{f}_{E}=\Upsilon\left(\chi, \delta_{e}\right)-\gamma\left(\chi, \delta_{e}^{*}\right)=\Xi\left(\chi, \delta_{e}^{*}\right)\left(\delta_{e}-\delta_{e}^{*}\right),
$$

where $\Xi(\cdot)=\Xi\left(\chi, \delta_{e}^{*}\right)=\partial \Upsilon(\chi, \varsigma) / \partial \varsigma \neq 0$ and $\varsigma=\vartheta_{h} \delta_{e}+$ $\left(1-\vartheta_{h}\right) \delta_{e}^{*}, \vartheta_{h} \in[0,1]$.

Then, Equation (47) can be converted to

$$
\dot{f}_{E}=\Xi\left(\chi, \delta_{e}^{*}\right) \delta_{e}-\Xi\left(\chi, \delta_{e}^{*}\right) \delta_{e}^{*}
$$

We employ the NN to approach $\delta_{e}^{*}$, the input vector is $\mathbf{X}_{h}=[\gamma, \theta, Q]^{T} \in \mathbf{R}^{3}$, and the ideal weight vector is $\mathbf{W}_{h}^{*}=$ $\left[w_{h 1}^{*}, w_{h 2}^{*}, \cdots, w_{h v_{1}}^{*}\right]^{T} \in \mathbf{R}^{v_{1}}$. The output form of the hidden layer is the same as Equation (23). $\varepsilon_{h} \in R$ and $\varepsilon_{h M} \in R^{+}$represent the approximation error and the upper bound of the approximation error, respectively. NN approximator can be expressed as

$$
\delta_{e}^{*}=\mathbf{W}_{h}^{* T} \phi_{h}\left(\mathbf{X}_{h}\right)+\varepsilon_{h},\left|\varepsilon_{h}\right| \leq \varepsilon_{h M} .
$$

We define $\varphi=\left\|\mathbf{W}_{h}^{*}\right\|^{2}$ and choose the following control law:

$$
\left\{\begin{array}{l}
\delta_{e}=\hbar(\vartheta)\left[\kappa_{h} f_{E}+\frac{\left(f_{E}\right) \widehat{\varphi} \phi_{h}^{T}\left(\mathbf{X}_{h}\right) \phi_{h}\left(\mathbf{X}_{h}\right)}{2}\right], \\
\hbar(\vartheta)=\exp \left(\vartheta^{2}\right) \cos \left(\frac{\pi \vartheta}{2}\right), \\
\vartheta=\kappa_{h}\left(f_{E}\right)^{2}+\frac{\left(f_{E}\right)^{2} \widehat{\varphi} \phi_{h}^{T}\left(\mathbf{X}_{h}\right) \phi_{h}\left(\mathbf{X}_{h}\right)}{2}
\end{array}\right.
$$

where $\kappa_{h}$ is a parameter to be designed. $\widehat{\varphi}$ is the estimation of $\varphi$, and the adaptive law is designed as

$$
\dot{\hat{\varphi}}=\frac{\omega_{h}}{2}\left(f_{E}\right)^{2} \phi_{h}^{T}\left(\mathbf{X}_{h}\right) \phi_{h}\left(\mathbf{X}_{h}\right)-2 \widehat{\varphi},
$$

where $\omega_{h} \in R^{+}$is the parameter to be designed.

Remark 14. Based on [36], we utilize the minimal learning parameter to define $\widehat{\varphi}$. In Equation (51), an overlarge $\omega_{h}$ in the adaptive law will depress the tracking performance at the transient, which should be designed appropriately. However, unlike $[20,36]$, the controller does not require high-order extended state observer with dynamic surface control; therefore, it reduces the amount of calculations.

\subsection{Stability Analysis of Altitude Subsystem}

Theorem 15. We consider the closed-loop altitude subsystem of HFV, under the premise of Assumption 11, consisting of nonaffine plant (42), control law (50), and adaptive law (51), and then, all the signals involved are bounded.

Proof. We define the estimation error as

$$
\tilde{\varphi}=\widehat{\varphi}-\varphi
$$

Select the following Lyapunov function as

$$
L_{h}=\frac{\left(f_{E}\right)^{2}}{2\left|\Xi\left(\chi, \delta_{\mathrm{e}}^{*}\right)\right|}+\frac{\tilde{\varphi}^{2}}{2 \omega_{h}} .
$$

Taking time derivative along (53), and substituting Equations (48), (49), and (51),

$$
\begin{aligned}
\dot{L}_{h}= & \frac{\left(f_{E}\right)\left(\dot{f}_{E}\right)}{\left|\Xi\left(\chi, \delta_{\mathrm{e}}^{*}\right)\right|}-\frac{\dot{\Xi}\left(\chi, \delta_{\mathrm{e}}^{*}\right)}{2\left|\Xi\left(\chi, \delta_{\mathrm{e}}^{*}\right)\right|^{2}}\left(f_{E}\right)^{2}+\frac{\tilde{\varphi} \dot{\hat{\varphi}}}{\omega_{h}} \\
= & \frac{\Xi\left(\chi, \delta_{\mathrm{e}}^{*}\right)\left(\delta_{\mathrm{e}}-\delta_{\mathrm{e}}^{*}\right) f_{E}}{\left|\Xi\left(\chi, \delta_{\mathrm{e}}^{*}\right)\right|}-\frac{\dot{\Xi}\left(\chi, \delta_{\mathrm{e}}^{*}\right)}{2\left|\Xi\left(\chi, \delta_{\mathrm{e}}^{*}\right)\right|^{2}}\left(f_{E}\right)^{2} \\
& +\frac{\tilde{\varphi}}{\omega_{h}}\left[\frac{\omega_{h}}{2}\left(f_{E}\right)^{2} \phi_{h}^{T}\left(\mathbf{X}_{h}\right) \phi_{h}\left(\mathbf{X}_{h}\right)-2 \widehat{\varphi}\right] \\
= & \frac{\Xi\left(\chi, \delta_{\mathrm{e}}^{*}\right)\left(\delta_{\mathrm{e}}-\mathbf{W}_{h}^{T} \phi_{h}\left(\mathbf{X}_{h}\right)+\varepsilon_{h M}\right) f_{E}}{\left|\Xi\left(\chi, \delta_{\mathrm{e}}^{*}\right)\right|} \\
& -\frac{\dot{\Xi}\left(\chi, \delta_{\mathrm{e}}^{*}\right)}{2\left|\Xi\left(\chi, \delta_{\mathrm{e}}^{*}\right)\right|^{2}}\left(f_{E}\right)^{2}-\frac{2 \widehat{\varphi} \tilde{\varphi}}{\omega_{h}}+\frac{\tilde{\varphi}}{2}\left(f_{E}\right)^{2} \phi_{h}^{T}\left(\mathbf{X}_{h}\right) \phi_{h}\left(\mathbf{X}_{h}\right) \\
= & \Theta \delta_{\mathrm{e}} f_{E}-\Theta \mathbf{W}_{h}^{T} \phi_{h}\left(\mathbf{X}_{h}\right) f_{E}+\Theta \varepsilon_{h M} f_{E}-\frac{\dot{\Xi}\left(\chi, \delta_{\mathrm{e}}^{*}\right)}{2\left|\Xi\left(\chi, \delta_{\mathrm{e}}^{*}\right)\right|^{2}}\left(f_{E}\right)^{2} \\
& -\frac{2 \widehat{\varphi} \tilde{\varphi}}{\omega_{h}}+\frac{\tilde{\varphi}}{2}\left(f_{E}\right)^{2} \phi_{h}^{T}\left(\mathbf{X}_{h}\right) \phi_{h}\left(\mathbf{X}_{h}\right),
\end{aligned}
$$

where $\Theta=\Xi\left(\chi, \delta_{\mathrm{e}}^{*}\right) /\left|\Xi\left(\chi, \delta_{\mathrm{e}}^{*}\right)\right| \in\{-1,1\}$.

Substituting Equation (50) and Equation (52) into Equation (54), we can get

$$
\begin{aligned}
\dot{L}_{h}= & \Theta \hbar(\vartheta)\left[\kappa_{h} f_{E}+\frac{\widehat{\varphi}}{2} f_{E} \phi_{h}^{T}\left(\mathbf{X}_{h}\right) \phi_{h}\left(\mathbf{X}_{h}\right)\right] f_{E}-\Theta f_{E} \mathbf{W}_{h}^{T} \phi_{h}\left(\mathbf{X}_{h}\right) \\
& +\Theta \varepsilon_{h M} f_{E}-\frac{\dot{\Xi}\left(\chi, \delta_{\mathrm{e}}^{*}\right)}{2\left|\Xi\left(\chi, \delta_{\mathrm{e}}^{*}\right)\right|^{2}}\left(f_{E}\right)^{2}+\frac{\left(f_{E}\right)^{2}}{2}(\widehat{\varphi}-\varphi) \phi_{h}^{T}\left(\mathbf{X}_{h}\right) \phi_{h}\left(\mathbf{X}_{h}\right) \\
& -\frac{2 \widehat{\varphi} \tilde{\varphi}}{\omega_{h}}=\Theta \hbar(\vartheta)\left[\kappa_{h}\left(f_{E}\right)^{2}+\frac{\widehat{\varphi}}{2}\left(f_{E}\right)^{2} \phi_{h}^{T}\left(\mathbf{X}_{h}\right) \phi_{h}\left(\mathbf{X}_{h}\right)\right] \\
& -\Theta f_{E} \mathbf{W}_{h}^{T} \phi_{h}\left(\mathbf{X}_{h}\right)+\Theta \varepsilon_{h M} f_{E}-\frac{\dot{\Xi}\left(\chi, \delta_{\mathrm{e}}^{*}\right)}{2\left|\Xi\left(\chi, \delta_{\mathrm{e}}^{*}\right)\right|^{2}}\left(f_{E}\right)^{2} \\
& +\frac{\left(f_{E}\right)^{2}}{2} \widehat{\varphi} \phi_{h}^{T}\left(\mathbf{X}_{h}\right) \phi_{h}\left(\mathbf{X}_{h}\right)-\frac{\left(f_{E}\right)^{2}}{2} \varphi \phi_{h}^{T}\left(\mathbf{X}_{h}\right) \phi_{h}\left(\mathbf{X}_{h}\right)-\frac{2 \widehat{\varphi} \tilde{\varphi}}{\omega_{h}}
\end{aligned}
$$


According to Equation (55),

$$
\begin{aligned}
\dot{L}_{h}= & -\kappa_{h}\left(f_{E}\right)^{2}+\kappa_{h}\left(f_{E}\right)^{2}+\frac{\left(f_{E}\right)^{2}}{2} \widehat{\varphi} \phi_{h}^{T}\left(\mathbf{X}_{h}\right) \phi_{h}\left(\mathbf{X}_{h}\right) \\
& +\Theta \hbar(\vartheta)\left[\kappa_{h}\left(f_{E}\right)^{2}+\frac{\widehat{\varphi}}{2}\left(f_{E}\right)^{2} \phi_{h}^{T}\left(\mathbf{X}_{h}\right) \phi_{h}\left(\mathbf{X}_{h}\right)\right] \\
& -\Theta f_{E} \mathbf{W}_{h}^{T} \phi_{h}\left(\mathbf{X}_{h}\right)+\Theta \varepsilon_{h M} f_{E}-\frac{\dot{\Xi}\left(\chi, \delta_{\mathrm{e}}^{*}\right)}{2\left|\Xi\left(\chi, \delta_{\mathrm{e}}^{*}\right)\right|^{2}}\left(f_{E}\right)^{2} \\
& -\frac{2 \widehat{\varphi} \tilde{\varphi}}{\omega_{h}}-\frac{\left(f_{E}\right)^{2}}{2} \varphi \phi_{h}^{T}\left(\mathbf{X}_{h}\right) \phi_{h}\left(\mathbf{X}_{h}\right) \\
= & -\left[\kappa_{h}+\frac{\dot{\Xi}\left(\chi, \delta_{\mathrm{e}}^{*}\right)}{\left.2\left|\Xi\left(\chi, \delta_{\mathrm{e}}^{*}\right)\right|^{2}\right]\left(f_{E}\right)^{2}+[1+\Theta \hbar(\vartheta)] \vartheta}\right. \\
& -\Theta f_{E} \mathbf{W}_{h}^{T} \phi_{h}\left(\mathbf{X}_{h}\right)+\Theta \varepsilon_{h M} f_{E}-\frac{2 \widehat{\varphi} \tilde{\varphi}}{\omega_{h}} \\
& -\frac{\left(f_{E}\right)^{2}}{2} \varphi \phi_{h}^{T}\left(\mathbf{X}_{h}\right) \phi_{h}\left(\mathbf{X}_{h}\right) .
\end{aligned}
$$

Owing to

$\tilde{\varphi}^{2}+2 \tilde{\varphi}(\widehat{\varphi}-\tilde{\varphi})+\varphi^{2}=\tilde{\varphi}^{2}+2 \tilde{\varphi} \varphi+\varphi^{2}=(\tilde{\varphi}+\varphi)^{2} \geq 0$,

we obtain

$$
2 \tilde{\varphi} \widehat{\varphi} \geq \tilde{\varphi}^{2}-\varphi^{2},
$$

by further noting that

$$
\begin{aligned}
-\Theta f_{E} \mathbf{W}_{h}^{T} \phi_{h}\left(\mathbf{X}_{h}\right) & = \pm f_{E} \mathbf{W}_{h}^{T} \phi_{h}\left(\mathbf{X}_{h}\right) \leq \frac{\left(f_{E}\right)^{2}}{2}\left\|\mathbf{W}_{h}^{T} \phi_{h}\left(\mathbf{X}_{h}\right)\right\|^{2}+\frac{1}{2} \\
& =\frac{\left(f_{E}\right)^{2}}{2}\left\|\mathbf{W}_{h}\right\|^{2}\left\|\phi_{h}\left(\mathbf{X}_{h}\right)\right\|^{2}+\frac{1}{2} \\
& =\frac{\left(f_{E}\right)^{2}}{2} \varphi \phi_{h}^{T}\left(\mathbf{X}_{h}\right) \phi_{h}\left(\mathbf{X}_{h}\right)+\frac{1}{2} .
\end{aligned}
$$

According to Young's inequality [37], the following inequality holds

$$
\begin{aligned}
\dot{L}_{h} \leq & -\left[\kappa_{h}+\frac{\dot{\Xi}\left(\chi, \delta_{\mathrm{e}}^{*}\right)}{2\left|\Xi\left(\chi, \delta_{\mathrm{e}}^{*}\right)\right|^{2}}\right]\left(f_{E}\right)^{2}+[1+\Theta \hbar(\vartheta)] \vartheta \\
& +\frac{\left(f_{E}\right)^{2}}{2} \varphi \phi_{h}^{T}\left(\mathbf{X}_{h}\right) \phi_{h}\left(\mathbf{X}_{h}\right)+\frac{1}{2}+\frac{\left(f_{E}\right)^{2}}{4}+\varepsilon_{h M}^{2} \\
& -\frac{\left(f_{E}\right)^{2}}{2} \varphi \phi_{h}^{T}\left(\mathbf{X}_{h}\right) \phi_{h}\left(\mathbf{X}_{h}\right)-\frac{\tilde{\varphi}^{2}-\varphi^{2}}{\omega_{h}} \\
= & -\left[\kappa_{h}+\frac{\dot{\Xi}\left(\chi, \delta_{\mathrm{e}}^{*}\right)}{2\left|\Xi\left(\chi, \delta_{\mathrm{e}}^{*}\right)\right|^{2}}-\frac{1}{4}\right]\left(f_{E}\right)^{2}-\frac{\tilde{\varphi}^{2}}{\omega_{h}}+\frac{\varphi^{2}}{\omega_{h}} \\
& +[1+\Theta \hbar(\vartheta)] \vartheta+\frac{1}{2}+\varepsilon_{h M}^{2} .
\end{aligned}
$$

TABLE 1: Initial trim conditions.

\begin{tabular}{lcc}
\hline Item & Value & Units \\
\hline$V$ & 2500 & $\mathrm{~m} / \mathrm{s}$ \\
$h$ & 27000 & $\mathrm{~m}$ \\
$\gamma$ & 0 & $\mathrm{deg}$ \\
$\theta$ & 1.5295 & $\mathrm{deg}$ \\
$Q$ & 0 & $\mathrm{deg} / \mathrm{s}$ \\
$\eta_{1}$ & 0.2857 & - \\
$\eta_{2}$ & 0.2857 & - \\
\hline
\end{tabular}

Let

$$
\kappa_{h}+\frac{\dot{\Xi}\left(\chi, \delta_{\mathrm{e}}^{*}\right)}{2\left|\Xi\left(\chi, \delta_{\mathrm{e}}^{*}\right)\right|^{2}}-\frac{1}{4}>0 .
$$

Then, we have

$$
\dot{L}_{h} \leq-l L_{h}+[1+\Theta \hbar(\vartheta)] \vartheta+\frac{1}{2}+\varepsilon_{h M}^{2}+\frac{\varphi^{2}}{\omega_{h}},
$$

with, $\iota=\min \left\{2\left|\Xi\left(\chi, \delta_{\mathrm{e}}^{*}\right)\right|\left[\kappa_{h}+\dot{\Xi}\left(\chi, \delta_{\mathrm{e}}^{*}\right) / 2\left|\Xi\left(\chi, \delta_{\mathrm{e}}^{*}\right)\right|^{2}-1 / 4\right]\right.$, $2\}$.

When $\dot{L}_{h} \leq 0$ if $L_{h} \geq\left([1+\Theta \hbar(\vartheta)] \vartheta+1 / 2+\varepsilon_{h M}^{2}\right) / \iota$.

Using the multiplication of Equation (62) by $e^{t t}$, it leads to

$$
\frac{\mathrm{d}}{\mathrm{d} t}\left(L_{h} e^{t t}\right) \leq-\iota L_{h}+[1+\Theta \hbar(\vartheta)] 9 e^{\imath t}+\left(\frac{1}{2}+\varepsilon_{h M}^{2}+\frac{\varphi^{2}}{\omega_{h}}\right) e^{t t} .
$$

Integrating Equation (63) over $[0, t]$, we have

$$
\begin{aligned}
L_{h}(t) \leq e^{-l t} \int_{0}^{t}[1+\Theta \hbar(9)] \vartheta e^{\imath \tau} \mathrm{d} \tau+\frac{1}{\iota}\left(\frac{1}{2}+\varepsilon_{h M}^{2}+\frac{\varphi^{2}}{\omega_{h}}\right) \\
+\left[L_{h}(0)-\frac{1}{\iota}\left(\frac{1}{2}+\varepsilon_{h M}^{2}+\frac{\varphi^{2}}{\omega_{h}}\right)\right] e^{-l t} .
\end{aligned}
$$

Based on Lemma 7, we know that $L_{h}$ is bounded. Meanwhile, $\tilde{\varphi}$ and $f_{E}$ are bounded. Since the polynomial $(s+r)^{3}$ is Hurwitz, the tracking error $\tilde{\gamma}$ is also bounded. By choosing an amply large $\kappa_{h}$ and sufficiently small $\omega_{h}$, the errors $\tilde{\gamma}, \tilde{\varphi}$, and $f_{E}$ can be arbitrarily small. Therefore, the closed-loop control system is locally uniformly asymptotically stable, and this is the end of the proof.

Remark 16. The proposed control scheme only requires one $\mathrm{NN}$ to approximate the uncertainty $\delta_{\mathrm{e}}^{*}$. By introducing the norm estimation approach [24], only one learning parameter 

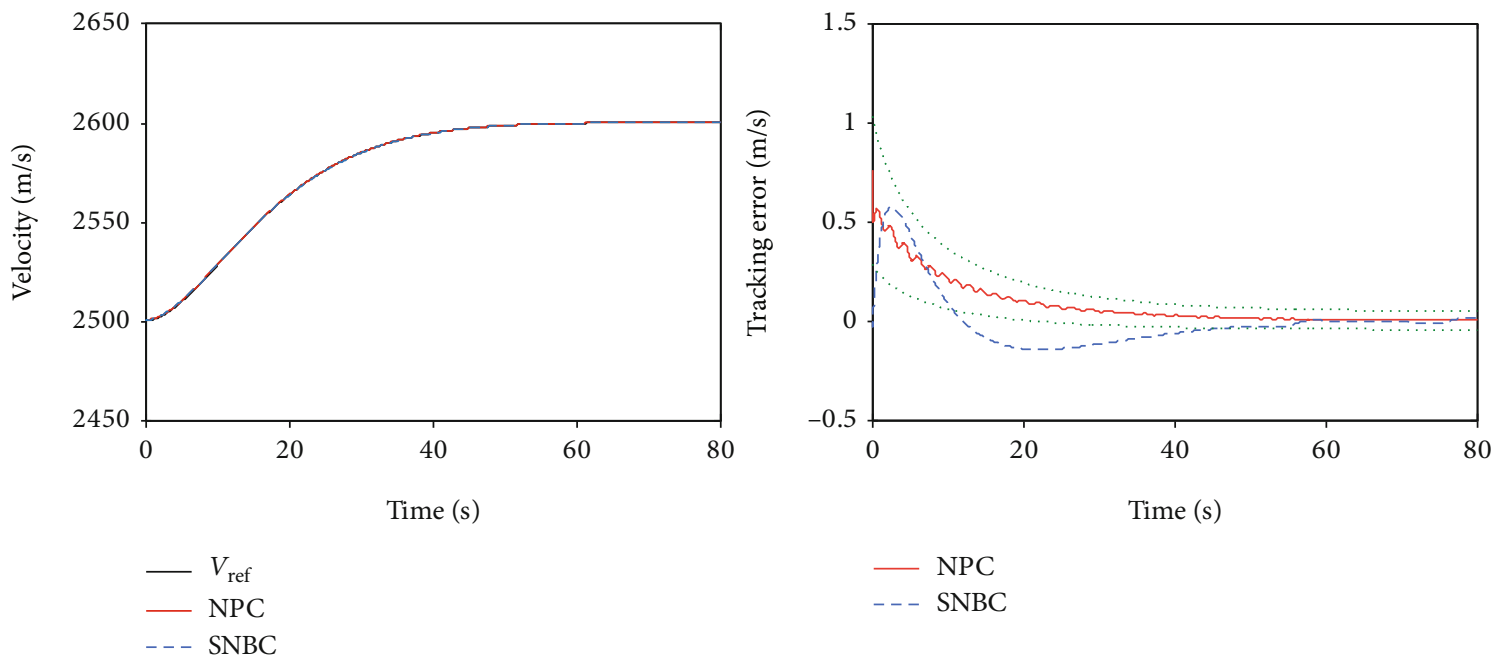

FIGURE 4: Velocity tracking performance of the case.
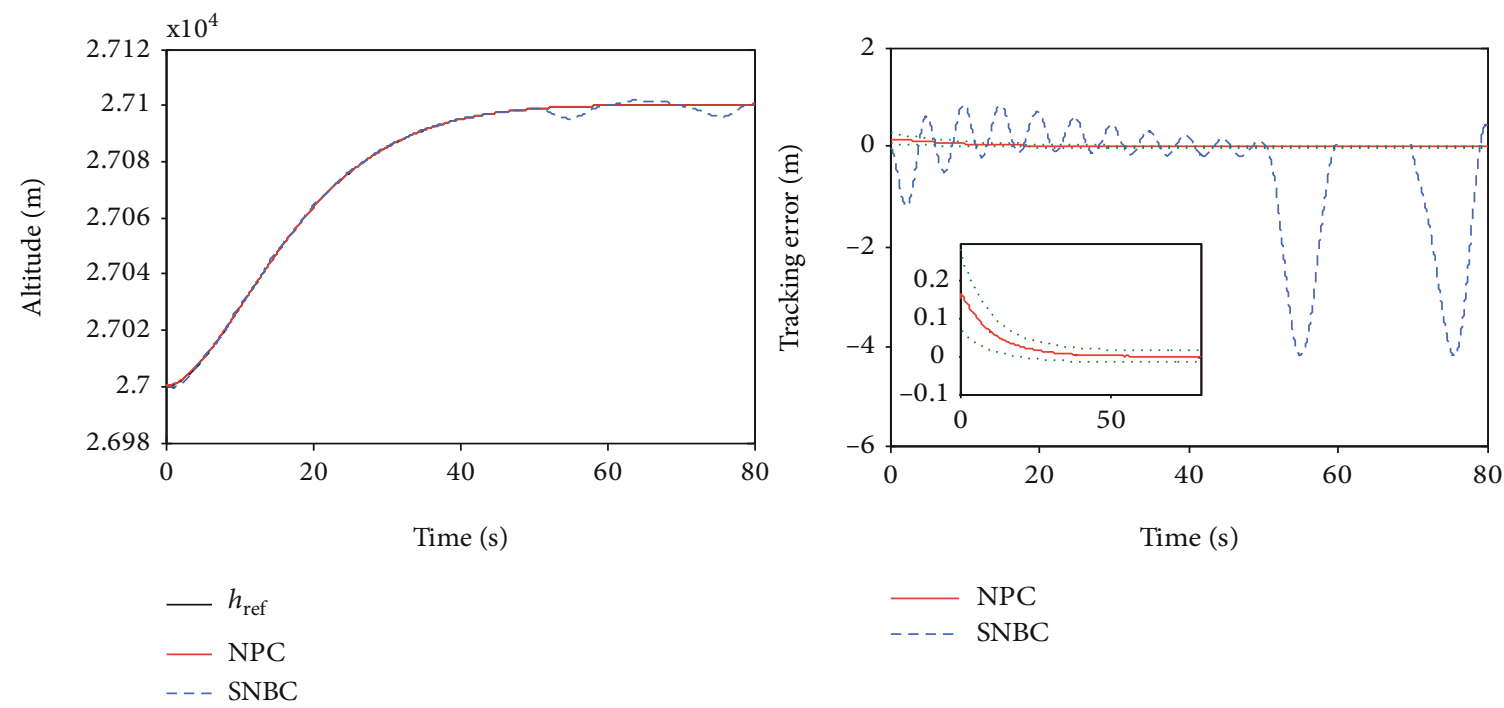

FIgURE 5: Altitude tracking performance of the case.

$\widehat{\varphi}$ is included in the NN approximator. Compared with [25], we do not require adjusting the elements of the weight vector online and needing the complex recursive processes based on back-stepping control either. Therefore, the amount of calculations in this paper is greatly reduced.

\section{Simulation Results}

To clarify and verify the performance of the proposed approximation-based prescribed performance control scheme, we present simulation studies in this section. The control object is the longitudinal model of HFV. The fourth-order Runge-Kutta method is used in the simulation, and the simulation step is $0.01 \mathrm{~s}$. The initial trim conditions of HFV are listed in Table 1, and aerodynamic coefficients and model parameters are borrowed from [27]. Both the velocity and altitude reference inputs are given by a secondorder reference model with a damping ratio of 0.9 and a nat- ural frequency of $0.1 \mathrm{rad} / \mathrm{s}$. The controller parameters are selected as $\lambda_{V 1}=0.3, \lambda_{V 2}=0.8, \omega_{h}=0.05, \lambda_{h}=2, \kappa_{h}=-25$, and $\ell=8$. The performance functions are designed as

$$
\left\{\begin{array}{l}
\lambda_{L}^{V}(t)=\left[\frac{\operatorname{sign}(\tilde{V}(0))-0.5}{\sinh (0.07 t+0.45)}\right]-0.15, \\
\lambda_{U}^{V}(t)=\left[\frac{\operatorname{sign}(\tilde{V}(0))+0.5}{\sinh (0.07 t+0.45)}\right]+0.15, \\
\lambda_{L}^{h}(t)=\left[\frac{\operatorname{sign}(\tilde{h}(0))-0.5}{\sinh (0.09 t+1.3)}\right]-0.05, \\
\lambda_{U}^{h}(t)=\left[\frac{\operatorname{sign}(\tilde{h}(0))+0.5}{\sinh (0.09 t+1.3)}\right]+0.05 .
\end{array}\right.
$$



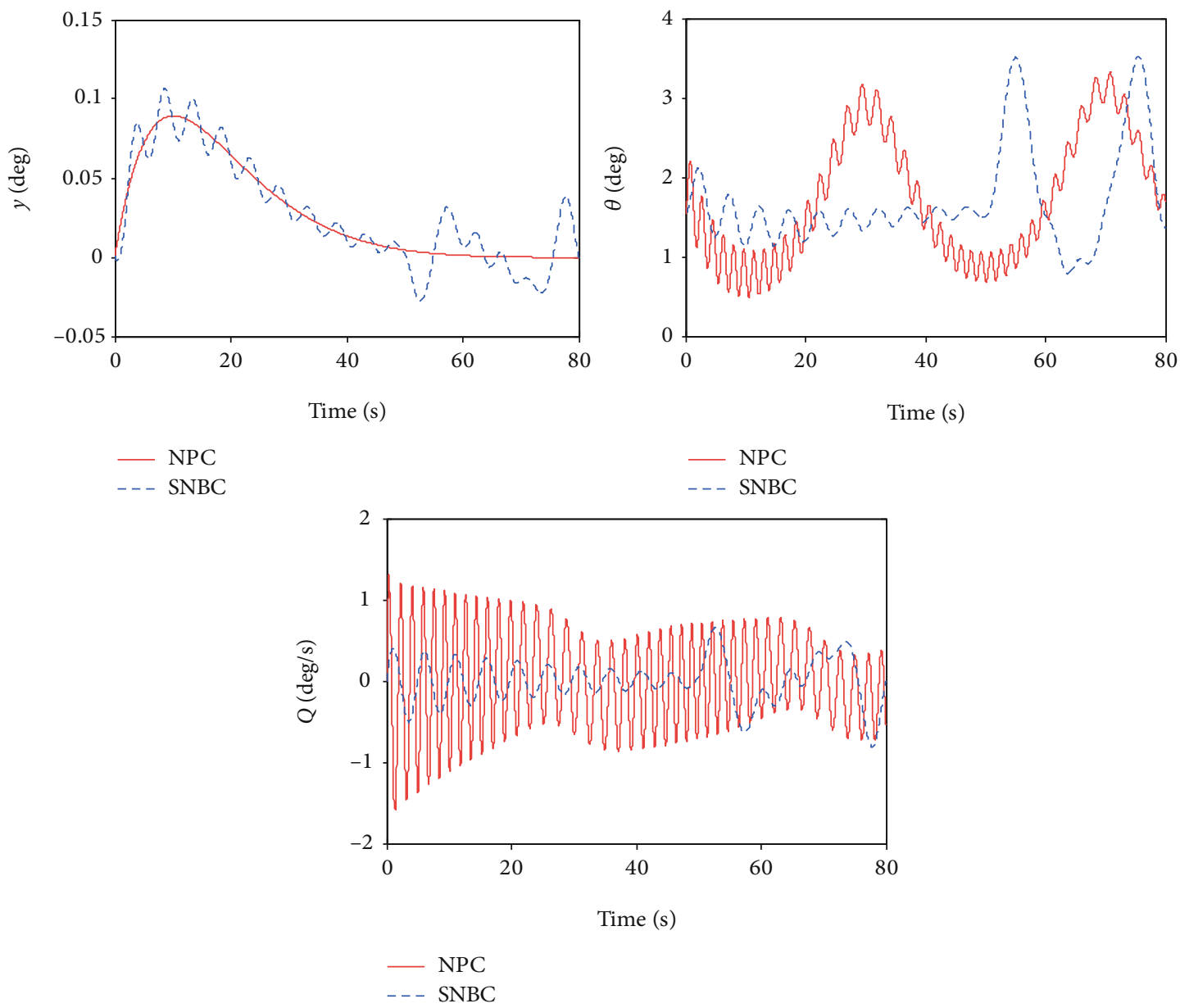

Figure 6: The attitude angles of the case.
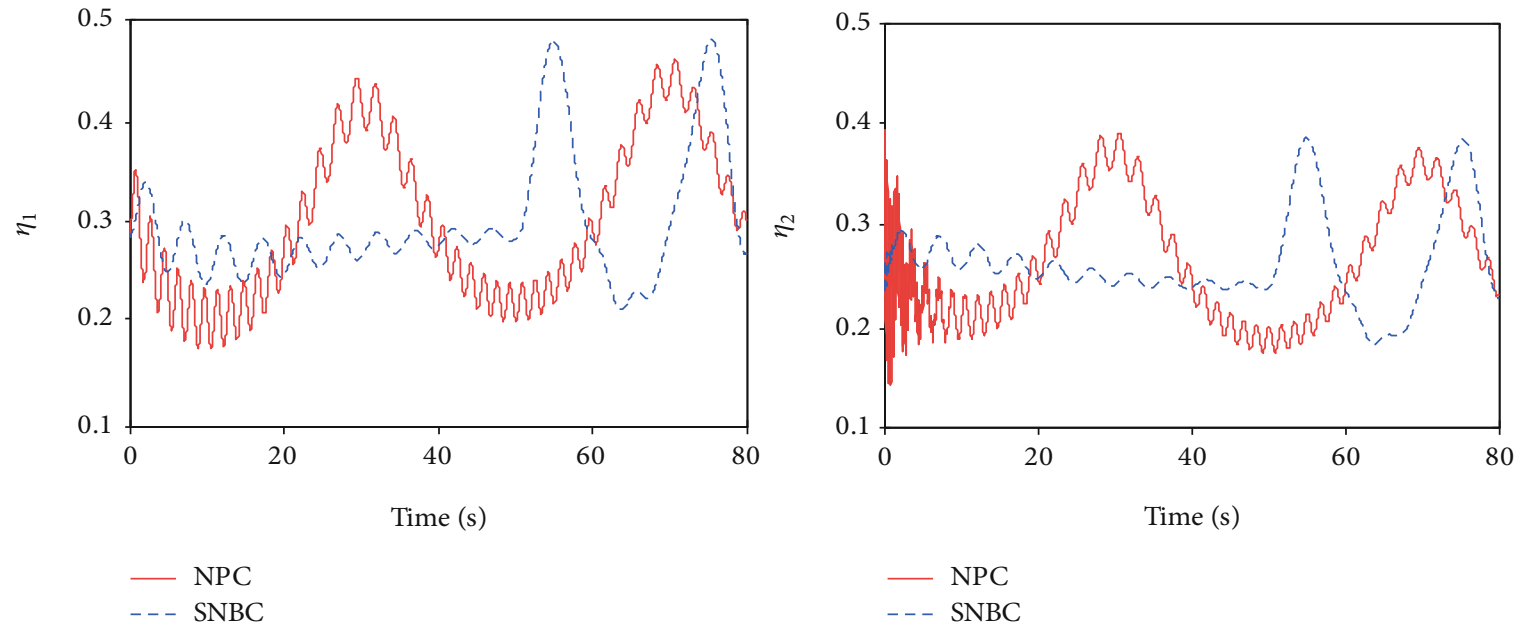

FIgURE 7: The flexible states of the case.

The input vector of the neural network is $\mathbf{X}_{h}=[\gamma, \theta, Q]^{T}$, with $\gamma=\left[-1^{\circ}, 1^{\circ}\right], \theta=\left[0^{\circ}, 5^{\circ}\right]$, and $Q=\left[-5^{\circ} / \mathrm{s}, 5^{\circ} / \mathrm{s}\right]$. The center vectors $\mathbf{c}_{1}$ and $\mathbf{c}_{2}$ are evenly spaced in their bounds. The number of nodes in the neural network is 20 . To show the superiority, the proposed novel prescribed performance con- trol (NPC) scheme is compared with a simplified neural back-stepping control (SNBC) strategy developed in [34].

Case 1 . The velocity tracks the step command with $100 \mathrm{~m} / \mathrm{s}$, and the altitude follows the step command with $100 \mathrm{~m}$. To test 


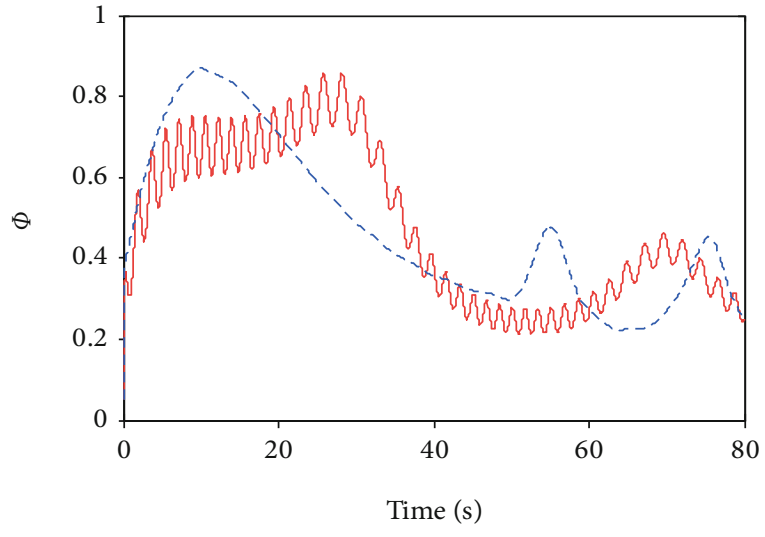

- NPC

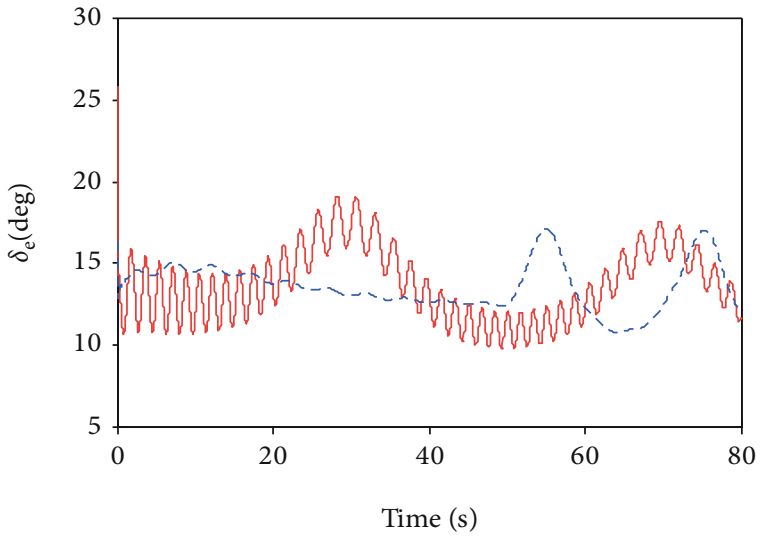

- NPC

- - SNBC

Figure 8: The control inputs of the case.
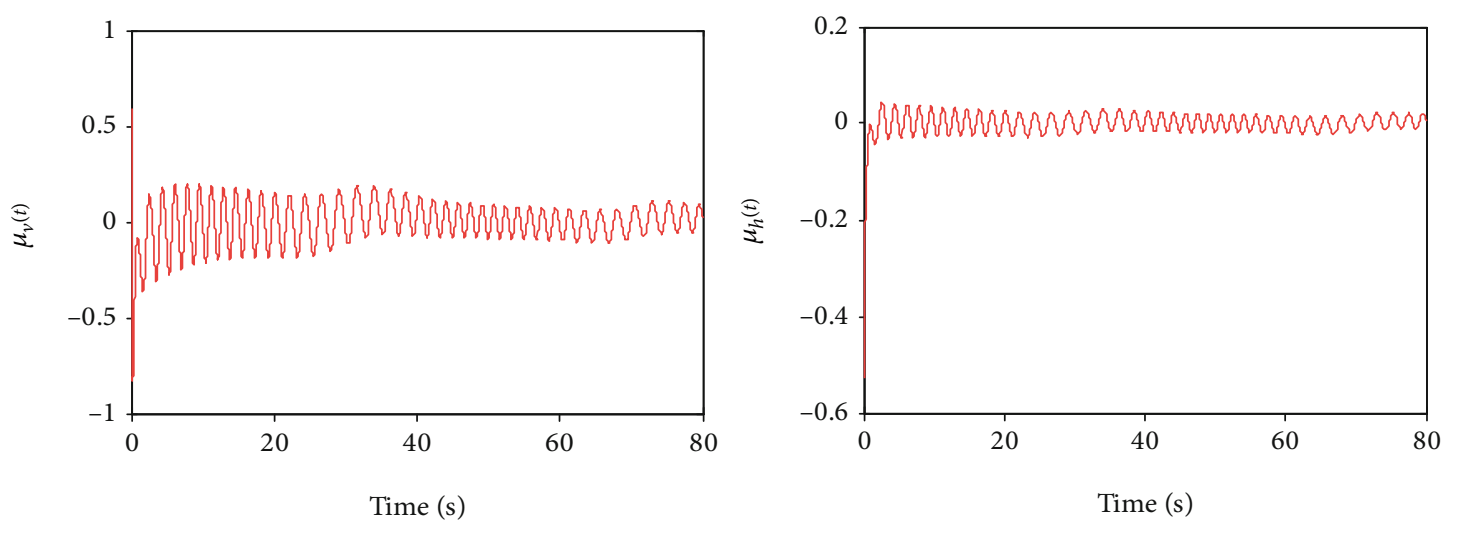

- NPC

Figure 9: $\mu_{V}(t)$ and $\mu_{h}(t)$ of the case.

the robustness of the proposed control strategy, all the aerodynamic coefficients are assumed to be uncertain. We define

$$
C= \begin{cases}C_{0}, & 0 \mathrm{~s} \leq t<40 \mathrm{~s}, \\ C_{0}[1+0.4 \sin (0.05 \pi t)], & 40 \mathrm{~s} \leq t<80 \mathrm{~s},\end{cases}
$$

where $C$ is the value of uncertain coefficient and $C_{0}$ denotes the normal value of $C$.

The obtained simulation results are depicted in Figures 4-10. It is apparent from Figures 4 and 5 that, compared with SNBC, the proposed NPC guarantees that velocity and altitude tracking errors are limited to the constructed prescribed behavior bounds with small overshoot and provide better velocity and altitude tracking performance in the presence of parametric uncertainties. For both control methodologies, attitude angles, flexible states, and control inputs, $\mu_{V}(t)$ and $\mu_{h}(t)$, shown in Figures 6 and 9, are bounded and smooth (without high-frequency chattering).

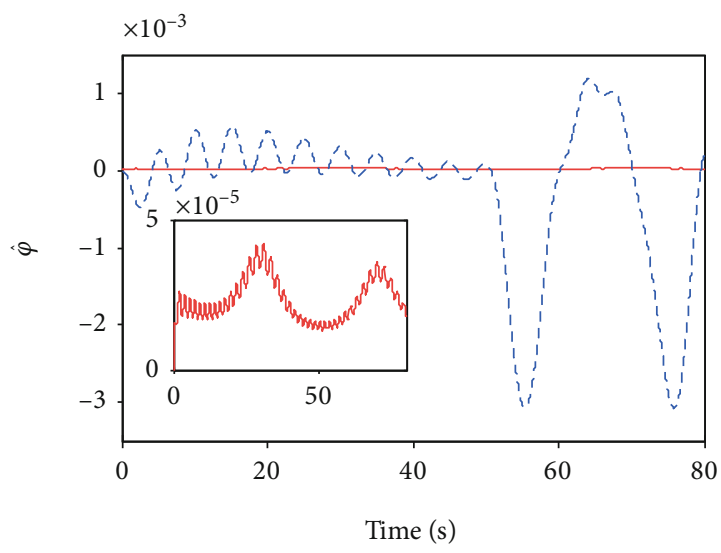

Figure 10: $\widehat{\varphi}$ of the case.

Figure 10 shows that $\widehat{\varphi}$ is bounded. To sum up, the superiority of the explored NPC over SNBC is well proven by the simulation results. 


\section{Conclusions}

In this paper, a novel neural approximation-based prescribed performance control scheme guaranteeing the tracking error with small, even zero, overshoot is proposed. New performance functions are exploited in the controller which can guarantee the velocity and altitude tracking errors with satisfactory transient and steady-state performance. And the new prescribed performance mechanism does not need to know the sign of the initial tracking error, and the shape of the functions can be changed according to the sign of the initial tracking error. The control scheme proposed in this paper eliminates the complex design process of backstepping. In addition, this paper uses only one neural network and advanced norm estimation approach, which reduces the complexity and computational pressure of the algorithm. Finally, the effectiveness and superiority of the proposed control strategy are validated by simulation results.

\section{Data Availability}

The data used to support the findings of this study are included within the article.

\section{Conflicts of Interest}

The authors declare no conflict of interest.

\section{Acknowledgments}

This research is financially supported by the Young Talent Support Project for Military Science and Technology under Grant No. 18-JCJQ-QT-007.

\section{References}

[1] H. An, Q. Wu, and H. Xia, "Adaptive controller design for a switched model of air-breathing hypersonic vehicles," Nonlinear Dynamics, vol. 94, no. 3, pp. 1851-1866, 2018.

[2] Q. Hu, Y. Meng, and C. Wang, "Adaptive backstepping control for air-breathing hypersonic vehicles with input nonlinearities," Aerospace Science and Technology, vol. 73, pp. 289299, 2018.

[3] X. Tao, Y. Jianqiang, and P. Zhiqiang, "State-estimator-integrated robust adaptive tracking control for flexible airbreathing hypersonic vehicle with noisy measurements," IEEE Transactions on Instrumentation and Measurement, vol. 99, pp. 1-15, 2019.

[4] C. Luo, H. Lei, D. Zhang, and X. Zou, "Adaptive neural control of hypersonic vehicles with actuator constraints," International Journal of Aerospace Engineering, vol. 2018, 15 pages, 2018.

[5] G. Gao, J. Wang, and X. Wang, "Robust tracking control for an air-breathing hypersonic vehicle with input constraints," International Journal of Systems Science, vol. 45, no. 12, pp. 2466-2479, 2014.

[6] X. Bu, X. Wu, R. Zhang, Z. Ma, and J. Huang, "Tracking differentiator design for the robust backstepping control of a flexible air-breathing hypersonic vehicle," Journal of the Franklin Institute, vol. 352, no. 4, pp. 1739-1765, 2015.
[7] W. Zhi, W. Bao, and H. Li, "Second-order dynamic slidingmode control for nonminimum phase underactuated hypersonic vehicles," IEEE Transactions on Industrial Electronics, vol. 64, no. 4, pp. 3105-3112, 2017.

[8] A. Hao, C. Wang, and B. Fidan, "Sliding mode disturbance observer-enhanced adaptive control for the air-breathing hypersonic flight vehicle," Acta Astronautica, vol. 139, pp. 111-121, 2017.

[9] X. Su and Y. Jia, "Self-scheduled robust decoupling control with $\mathrm{H} \infty \mathrm{o}$ performance of hypersonic vehicles," Systems \& Control Letters, vol. 70, pp. 38-48, 2014.

[10] G. Wu and X. Meng, "Nonlinear disturbance observer based robust backstepping control for a flexible air-breathing hypersonic vehicle," Aerospace Science and Technology, vol. 54, pp. 174-182, 2016.

[11] B. Xu, X. Huang, and D. Wang, "Dynamic surface control of constrained hypersonic flight models with parameter estimation and actuator compensation," Asian Journal of Control, vol. 16, no. 1, pp. 162-174, 2014.

[12] H. Sun, S. Li, and C. Sun, "Finite time integral sliding mode control of hypersonic vehicles," Nonlinear Dynamics, vol. 73, no. 1-2, pp. 229-244, 2013.

[13] J. Wang, Q. Zong, B. Tian, and H. Liu, "Flight control for a flexible air-breathing hypersonic vehicle based on quasicontinuous high-order sliding mode," Journal of Systems Engineering and Electronics, vol. 24, no. 2, pp. 288-295, 2013.

[14] B. Tian, R. Su, and W. Fan, "Multiple time scale smooth second order sliding mode controller design for flexible hypersonic vehicles," Proceedings of the Institution of Mechanical Engineers Part G: Journal of Aerospace Engineering, vol. 229, no. 5, pp. 781-791, 2015.

[15] P. Wang, J. Wang, X. Bu, C. Luo, and S. Tan, “Adaptive fuzzy back-stepping control of a flexible air-breathing hypersonic vehicle subject to input constraints," Journal of Intelligent \& Robotic Systems, vol. 87, no. 3-4, pp. 565-582, 2017.

[16] C. Guo, X. Liang, J. Wang, and $\mathrm{H}$. Wu, "Mixed $\mathrm{H}_{2} / \mathrm{H}_{\infty}$ decentralized fuzzy tracking control design for a flexible airbreathing hypersonic vehicle," Proceedings of the Institution of Mechanical Engineers, Part I: Journal of Systems and Control Engineering, vol. 229, no. 5, pp. 388-405, 2014.

[17] C. P. Bechlioulis and G. A. Rovithakis, "Robust adaptive control of feedback linearizable MIMO nonlinear systems with prescribed performance," IEEE Transactions on Automatic Control, vol. 53, no. 9, pp. 2090-2099, 2008.

[18] C. P. Bechlioulis and G. A. Rovithakis, "Adaptive control with guaranteed transient and steady state tracking error bounds for strict feedback systems," Automatica, vol. 45, no. 2, pp. 532-538, 2009.

[19] L. Zhang, S. Tong, and Y. Li, "Prescribed performance adaptive fuzzy output-feedback control of uncertain nonlinear systems with unmodeled dynamics," Nonlinear Dynamics, vol. 77, no. 4, pp. 1653-1665, 2014.

[20] X. Shao, L. Wang, J. Li, and J. Liu, "High-order ESO based output feedback dynamic surface control for quadrotors under position constraints and uncertainties," Aerospace Science and Technology, vol. 89, pp. 288-298, 2019.

[21] X. Bu, X. Wu, F. Zhu, J. Huang, Z. Ma, and R. Zhang, "Novel prescribed performance neural control of a flexible airbreathing hypersonic vehicle with unknown initial errors," ISA Transactions, vol. 59, pp. 149-159, 2015. 
[22] Z. Guan, Y. Ma, Z. Zheng, and N. Guo, "Prescribed performance control for automatic carrier landing with disturbance," Nonlinear Dynamics, vol. 94, no. 2, pp. 1335-1349, 2018.

[23] X. Bu, X. Wu, R. Zhang, Z. Ma, and J. Huang, "A neural approximation-based novel back-stepping control scheme for air-breathing hypersonic vehicles with uncertain parameters," Proceedings of the Institution of Mechanical Engineers, Part I: Journal of Systems and Control Engineering, vol. 230, no. 3, pp. 231-243, 2016.

[24] C. Wang and Y. Lin, "Multivariable adaptive backstepping control: a norm estimation approach," IEEE Transactions on Automatic Control, vol. 57, no. 4, pp. 989-995, 2012.

[25] X. Bu, X. Wu, Z. Ma, and R. Zhang, "Nonsingular direct neural control of air-breathing hypersonic vehicle via back-stepping," Neurocomputing, vol. 153, pp. 164-173, 2015.

[26] M. A. Bolender and D. B. Doman, "Nonlinear longitudinal dynamical model of an air-breathing hypersonic vehicle," Journal of Spacecraft and Rockets, vol. 44, no. 2, pp. 374-387, 2007.

[27] J. T. Parker, A. Serrani, and S. Yurkovich, "Control-oriented modeling of an air-breathing hypersonic vehicle," Journal of Guidance Control \& Dynamics, vol. 30, no. 3, pp. 856-869, 2007.

[28] Y. Wang, J. Hu, J. Li, and B. Liu, "Improved prescribed performance control for nonaffine pure-feedback systems with input saturation," International Journal of Robust and Nonlinear Control, vol. 29, no. 6, pp. 1769-1788, 2019.

[29] R. M. Sanner and J. J. E. Slotine, "Gaussian networks for direct adaptive control," IEEE Transactions on Neural Networks, vol. 3, no. 6, pp. 837-863, 1992.

[30] X. Shao, B. Tian, W. Yang, and W. Zhang, "Estimator-based MLP neuroadaptive dynamic surface containment control with prescribed performance for multiple quadrotors," Aerospace Science and Technology, vol. 97, article 105620, 2020.

[31] R. D. Nussbaum, "Some remarks on a conjecture in parameter adaptive control," Systems \& Control Letters, vol. 3, no. 5, pp. 243-246, 1983.

[32] C. P. Bechlioulis and G. A. Rovithakis, "A low-complexity global approximation-free control scheme with prescribed performance for unknown pure feedback systems," Automatica, vol. 50, no. 4, pp. 1217-1226, 2014.

[33] Q. Zong, F. Wang, and B. Tian, "Robust adaptive dynamic surface control design for a flexible air-breathing hypersonic vehicle with input constraints and uncertainty," Nonlinear Dynamics, vol. 78, no. 1, pp. 289-315, 2014.

[34] X. Bu, X. Wu, J. Huang, Z. Ma, and R. Zhang, "Minimal-learning-parameter based simplified adaptive neural back-stepping control of flexible air-breathing hypersonic vehicles without virtual controllers," Neurocomputing, vol. 175, pp. 816-825, 2016.

[35] X. Shao, J. Liu, H. Cao, and C. Shen, "Robust dynamic surface trajectory tracking control for a quadrotor UAV via extended state observer," International Journal of Robust and Nonlinear Control, vol. 28, no. 7, pp. 2700-2719, 2018.

[36] X. Shao and Y. Shi, "Neural adaptive control for MEMS gyroscope with full-state constraints and quantized input," IEEE Transactions on Industrial Informatics, vol. 16, no. 10, pp. 6444-6454, 2020.

[37] H. Wang, X. Li, and K. Liu, "Adaptive neural control for a general class of pure-feedback stochastic nonlinear systems," Neurocomputing, vol. 135, pp. 348-356, 2014. 